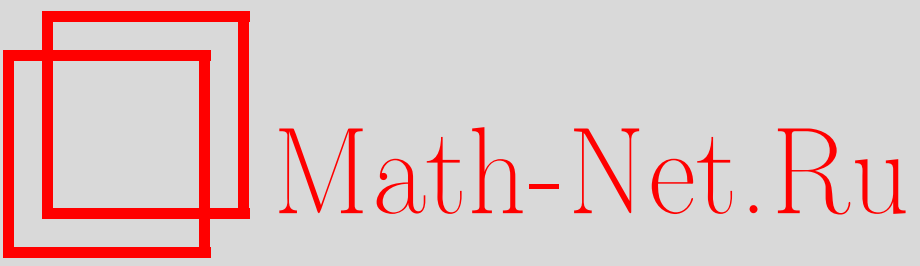

А. Л. Якымив, Асимптотика на бесконечности отрицательно биномиально безгранично делимых распределений, Теория вероятн. и ее примен., 2010, том 55, выпуск 2, 373-382

DOI: https://doi.org/10.4213/tvp4209

Использование Общероссийского математического портала Math-Net.Ru подразумевает, что вы прочитали и согласны с пользовательским соглашением

http://www . mathnet.ru/rus/agreement

Параметры загрузки:

IP : 3.93 .64 .190

26 апреля 2023 г., 16:35:00 
(C) $2010 \Gamma$

ЯКЫМив А. Л.*

\section{АСИМПТОТИКА НА БЕСКОНЕЧНОСТИ ОТРИЦАТЕЛЬНО БИНОМИАЛЬНО БЕЗГРАНИЧНО ДЕЛИМЫХ РАСПРЕДЕЛЕНИЙ}

Согласно С. Янкович (Publ. Inst. Math. (Beograd), 1993, v. 54, p. 126$134)$, случайная величина $Y$ имеет отрицательно биномиально безгранично делимое распределение тогда и только тогда, когда ее характеристическая функция $\varphi(t)$ допускает представление

$$
\varphi(t)=\frac{1}{(1-\ln \psi(t))^{r}}
$$

для некоторых $r>0$ и безгранично делимой характеристической функции $\psi(t)$. В настоящей статье для некоторого класса случайных величин $Y$ с отрицательно биномиально безгранично делимым распределением получена асимптотика $\mathbf{P}\{Y>t\}$ при $t \rightarrow \infty$, выраженная в терминах спектральной меры представления Леви безгранично делимой характеристической функции $\psi(t)$.

Ключевые слова и фразы: отрицательно биномиально безгранично делимое распределение, характеристическая функция, спектральная мера Леви, преобразование Лапласа, слабо осциллирующие функции, мажорируемо меняющиеся функции, слабая эквивалентность функций на бесконечности.

1. Введение. Предположим, что случайная величина $\nu_{p}^{(r)}$ имеет отрицательное биномиальное распределение:

$$
\mathbf{P}\left\{\nu_{p}^{(r)}=n\right\}=C_{-r}^{n} p^{r}(-q)^{n},
$$

$n=0,1,2 \ldots, p>0, p+q=1, r>0$, где

$$
C_{-r}^{n}=\frac{(-r)(-r-1) \cdots(-r-n+1)}{n !}
$$

при $n \geqslant 1$ и $C_{-r}^{0}=1$. Согласно [7], случайная величина $Y$ имеет отрицательно биномиально безгранично делимое распределение, если для каждого $p \in(0,1)$ существует последовательность независимых одинаково распределенных случайных величин $X_{p}^{(1)}, X_{p}^{(2)}, \ldots$, не зависящих от $\nu_{p}^{(r)}$, таких, что

$$
Y \stackrel{d}{=} \lim _{p \rightarrow 0} \sum_{j=1}^{\nu_{p}^{(r)}} X_{p}^{(j)}
$$

(последний предел понимается в слабом смысле). Как показано в [7], случайная величина $Y$ имеет отрицательно биномиально безгранично делимое распределение тогда и только тогда, когда ее характеристическая функция $\varphi(t)$ представима в виде

$$
\varphi(t)=\frac{1}{(1-\ln \psi(t))^{r}}, \quad t \in \mathbf{R}^{1}
$$

* Математический институт им. В.А.Стеклова РАН, ул. Губкина, 8, 119991 Москва, Россия; e-mail: arsen@mi.ras.ru

1) Работа выполнена при поддержке Российского фонда фундаментальных исследований (грант № 08-01-00563) и программы «Ведущие научные школы» (грант НШ4129.2006.1). 
где $\psi(t)$ является характеристической функцией некоторой безгранично делимой случайной величины $Z$, т.е.

$$
\psi(t) \equiv \mathbf{E} e^{i t Z}=\exp \left(i \alpha t-\frac{\sigma^{2} t^{2}}{2}+\int_{-\infty}^{\infty}\left(e^{i t x}-1-\frac{i t x}{1+x^{2}}\right) G(d x)\right),
$$

где $G(d x)$ - ее спектральная мера Леви [8, раздел 2.2 , соотношение $(2.12)]$ на $(-\infty, 0) \cup$ $(0, \infty)$ (возможно, неограниченная в окрестности нуля), причем конечны интегралы

$$
\int_{-1}^{1} x^{2} G(d x), \quad \int_{-\infty}^{-1} G(d x), \quad \int_{1}^{\infty} G(d x)
$$

$\sigma \geqslant 0, \alpha-$ некоторое действительное число, $t \in(-\infty, \infty)$.

Отметим два важных частных случая (1). Если $r=1$, то мы получаем известный случай геометрически безгранично делимого распределения (см. статью [9] и книгу [10]). Если $r$ - произвольное натуральное число, то мы приходим к случаю Паскаля безгранично делимого распределения [7].

Мы изучаем асимптотику на бесконечности $\mathbf{P}\{Y>t\}$, выраженную в терминах спектральной функции

$$
q(t)=\int_{t}^{\infty} G(d x), \quad t>0 .
$$

В случае безгранично делимых распределений аналогичная задача была поставлена и частично решена в 1961 г. В. М. Золотаревым в [6]. К настоящему моменту имеется большое число публикаций в этом направлении (см. ссылки в статьях [1], [2], [12] и книгах [11] и [15]). В частности, в [15] представлены некоторые результаты относительно вероятностей больших уклонений случайной величины $Z$, полученные при помощи тауберовых теорем. При проверке соответствующих тауберовых условий в [15] существенно использовался тот факт, что $\alpha$ является параметром сдвига для случайной величины $Z$, слагаемое $\sigma^{2} t^{2} / 2$ дает ее нормальную компоненту, а разложение меры $G(d x)$ в виде суммы двух мер дает свертку соответствующих распределений. Однако, как нетрудно заметить, ни то, ни другое, ни третье не справедливо для случайной величины $Y$. Поэтому в данном случае не удается свести задачу к исследованию асимптотики на бесконечности неотрицательной случайной величины с параметрами $\alpha=\sigma=0$, как это было сделано в [15].

В настоящей статье используется другой подход, основанный на рассмотрении двусторонних преобразований Лапласа меры $G(d x)$ и распределения случайной величины $Y$ в предположении, что они существуют на некотором непустом интервале действительной прямой. Далее проводится асимптотический анализ соотношений для $n$-й производной этих преобразований с последующим применением тауберовой теоремы. В частности, показано, что если $q(t+u) / q(t) \rightarrow 1$ и $u=o(t)$ при $t \rightarrow \infty$, то

$$
\mathbf{P}\{Y>t\}=(1+o(1)) r q(t) .
$$

\section{2. Основной результат.}

О п р е д е л е н и е $1[15$, определение 1.6]. Пусть функции $f(t)$ и $g(t)$ заданы и положительны при $t \geqslant a \geqslant 0$. Мы будем говорить, что $f(t)$ и $g(t)$ слабо асимптотически эквивалентны при $t \rightarrow \infty$ и писать

$$
f(t) \stackrel{w}{\sim} g(t)
$$

если для произвольного $\varepsilon$ можно указать такое $\delta_{0} \in(0,1)$, что для любого $\delta \in\left(0, \delta_{0}\right)$ найдется $t_{0}>0$ такое, что при $t \geqslant t_{0}$ выполнены неравенства

$$
(1-\varepsilon) g(t(1+\delta)) \leqslant f(t) \leqslant(1+\varepsilon) g(t(1-\delta)) .
$$

Основным результатом настоящей статьи является следующая теорема. 
Теорема 1. Предположим, что для некоторого $а>0$ при $\lambda \in(0, a)$ выполнено

$$
-\infty<\int_{\mathbf{R}^{1}}\left(e^{-\lambda x}-1\right) G(d x)<\infty
$$

где $G(d x)$ - спектральная мера Леви характеристической функиии $\psi(t)($ cм. (2)). Пусть «хвост» спектральной меры $G(d x)$ - функиия $q(t)$, определяемая соотношением (3) - мажорируемо меняется на бесконечности [13, приложение П.3], т.е.

$$
\limsup _{t \rightarrow \infty} \frac{q(t)}{q(2 t)}<\infty
$$

Тогда

$$
\mathbf{P}\{Y>t\} \stackrel{w}{\sim} r q(t) \quad \text { npu } t \rightarrow \infty .
$$

Следствие 1. Пусть выполнено (4) и функиия $q(t)$ слабо осииллирует на бесконечности [15, раздел 1.4], т.е. $q(t+u) / q(t) \rightarrow 1 u u=o(t)$ nрu $t \rightarrow \infty$. Тогда

$$
\mathbf{P}\{Y>t\}=(1+o(1)) r q(t) .
$$

Отметим, в частности, что всякая правильно меняющаяся функция $q(t)$ является слабо осциллирующей, т.е. для нее выполнено (6), если имеет место (4). В книге автора [15, раздел 1.4] приведен широкий класс примеров слабо осциллирующих функций, не являющихся правильно меняющимися. В работах [3], [4] слабо осциллирующие функции изучались в связи с другими теоретико-вероятностными приложениями. В [4] содержится подробная библиография по этому поводу.

Пусть $f(t)$ - положительная функция, определенная при $t \in \mathbf{R}^{1}$, и $F(d x)-\sigma$ конечная мера на $\mathbf{R}^{1}$. Мы будем полагать

$$
\begin{gathered}
\widehat{f}(\lambda)=\int_{0}^{\infty} e^{-\lambda x} f(x) d x, \quad \bar{f}(\lambda)=\int_{-\infty}^{0} e^{-\lambda x} f(x) d x, \\
\widetilde{F}(\lambda)=\int_{\mathbf{R}^{1}} e^{-\lambda x} F(d x)
\end{gathered}
$$

в предположении, что последние интегралы существуют на некотором непустом интервале действительной прямой. В дополнение к (3) положим

$$
q(t)=\int_{-\infty}^{t} G(d x), \quad t<0
$$

Сформулируем несколько лемм.

Лемма 1. Пусть выполнено условие (4). Тогда у характеристической функиии $\psi(t)$ из (2) существует аналитическое продолжение на полосу $\{t: t=\nu+i \lambda(\nu \in$ $\left.\left.\mathbf{R}^{1}, \lambda \in(0, a)\right)\right\}$, причем $\ln \psi(i \lambda)$ представима при $\lambda \in(0, a)$ в виде

$$
\ln \psi(i \lambda)=-\beta \lambda+\frac{\sigma^{2} \lambda^{2}}{2}+\lambda \bar{q}(\lambda)-\lambda \widehat{q}(\lambda),
$$

где $\bar{q}(\lambda)$ u $\widehat{q}(\lambda)$ конечнь при $\lambda \in(0, a)$ (см. обозначения (3), (7) u (8)), а $\beta$ - некоторое действительное число.

Пусть $f(t) n$ раз дифференцируема в точке $t$. Мы положим

$$
f^{(n)}(t)=\frac{d^{n}}{d t^{n}} f(t)
$$

при $n \in \mathbf{N}$ и $f^{(n)}(t)=f(t)$ при $n=0$. Также положим

$$
I_{n}=\int_{0}^{\infty} t^{n} q(t) d t
$$

и, согласно лемме 1 ,

$$
g(\lambda)=-\frac{\ln \psi(i \lambda)}{\lambda}=\beta-\frac{\sigma^{2} \lambda}{2}-\bar{q}(\lambda)+\widehat{q}(\lambda), \quad \lambda \in(0, a)
$$


Лемма 2. Пусть выполнень все предположения теоремь 1. Тогда для произвольного фиксированного неотричательного челого числа $\mathrm{n}$ пр $\lambda \rightarrow 0_{+}$

$$
g^{(j)}(\lambda)=O\left(\left|\widehat{q}^{(j)}(\lambda)\right|\right)
$$

Лемма 3. Пусть функиия $q(t)$ мажорируемо меняется на бесконечности (т.е. выполнено (5)). Тогда для всех неотрицательньх иельх $i, j$ при $\lambda \rightarrow 0_{+}$

$$
\widehat{q}^{(i)}(\lambda) \widehat{q}^{(j)}(\lambda)=O\left(\left|\widehat{q}^{(i+j+1)}(\lambda)\right|\right),
$$

причем символ «O» в последнем выражении можно заменить на «о», если $I_{i+j+1}=$ $\infty$.

Лемма 4. Пусть $n \in \mathbf{N}$, выполнены все предположения теоремы 1 и набор неотрицательньх чельх чисел $i_{1}, \ldots, i_{n}$ таков, ито

$$
\sum_{j=1}^{n} j i_{j}=n
$$

Тогда при $\lambda \rightarrow 0_{+}$

$$
\prod_{j=1}^{n}\left((\lambda g(\lambda))^{(j)}\right)^{i_{j}}=O\left(\left|\widehat{q}^{(n-1)}(\lambda)\right|\right),
$$

причем символ «О» в последнем выражении можно заменить на «о», если $i_{n}=0$ и $I_{n-1}=\infty$.

Лемма 5. Пусть функиии $f(t)$ и $g(t)$ определень, положительньи и не возрастают при $t \in \mathbf{R}_{+}^{1}$,

$$
\limsup _{t \rightarrow \infty} \frac{g(t)}{g(2 t)}<\infty
$$

и существует такое $M$, что для каждого фиксированного $n \geqslant M$

Тогда

$$
\frac{d^{n}}{d \lambda^{n}} \widehat{f}(\lambda)=(1+o(1)) \frac{d^{n}}{d \lambda^{n}} \widehat{g}(\lambda) \quad\left(\lambda \rightarrow 0_{+}\right)
$$

$$
f(t) \stackrel{w}{\sim} g(t) \quad(t \rightarrow \infty)
$$

Пусть $\left(U_{k}, k \in \mathbf{N}\right)$ - последовательность $\sigma$-конечных мер на $\mathbf{R}^{1}$. Следуя $[1$, раздел VIII.6], мы будем говорить, что последовательность $\left(U_{k}, k \in \mathbf{N}\right)$ слабо сходится к $\sigma$-конечной мере $U$ на $\mathbf{R}^{1}$, и писать

$$
U_{k} \Rightarrow U \quad(k \rightarrow \infty),
$$

если $U_{k}(A) \rightarrow U(A)$ для каждого ограниченного борелевского множества $A \subseteq \mathbf{R}^{1}$ такого, что $U(\partial A)=0$.

Лемма 6. Пусть для мер $U$ и $V$ существуют их двусторонние преобразования Лапласа $($ см. (7)) $\widetilde{U}(\lambda)$ и $\widetilde{V}(\lambda)$ при $\lambda \in(a, b)$ для некоторых $a, b, 0<a<b<\infty$, причем $\widetilde{U}(\lambda)=\widetilde{V}(\lambda)$ для любого $\lambda \in(a, b)$. Тогда $U=V$.

Лемма 7. Пусть для последовательности ( $\left.U_{k}, k \in \mathbf{N}\right)$ при некоторых $a, b$, $0<a<b<\infty$, существуют (двусторонние) преобразования Лапласа:

1) Eсли при $k \rightarrow \infty$

$$
\widetilde{U}_{k}(\lambda)=\int_{\mathbf{R}^{1}} e^{-\lambda x} U_{k}(d x)<\infty \quad \forall \lambda \in(a, b) .
$$

$$
\widetilde{U}_{k}(\lambda) \rightarrow \omega(\lambda)<\infty \quad \forall \lambda \in(a, b),
$$

то $\omega(\lambda)$ является преобразованием Лапласа некоторой меры $U$ на $\mathbf{R}^{1}$ и $U_{k} \Rightarrow U$ при $k \rightarrow \infty$.

2) Обратно, если $U_{k} \Rightarrow U$ при $k \rightarrow \infty u \widetilde{U}_{k}(a)$ и $\widetilde{U}_{k}(b)$ ограничены, то выполнено соотношение (11) и для мерь $U$ существует преобразование Лапласа $\widetilde{U}(\lambda)=\omega(\lambda)$ прu $\lambda \in(a, b)$. 
3. Доказательства. Д о к а з а т е ль с т в о л е м м ы 1 . Поскольку выполнено (4), то $\int_{-1}^{1} x G(d x)<\infty$, и для некоторого действительного $\beta$

$$
\ln \psi(t)=i \beta t-\frac{\sigma^{2} t^{2}}{2}+\int_{-\infty}^{\infty}\left(e^{i t x}-1\right) G(d x),
$$

$t \in \mathbf{R}^{1}$. Далее, в силу (4) существует аналитическое продолжение интеграла $\int_{-\infty}^{\infty}\left(e^{i t x}-1\right) G(d x)$ на полосу $t=\nu+i \lambda\left(\nu \in \mathbf{R}^{1}, \lambda \in(0, a)\right)$. Поэтому при $\lambda \in(0, a)$

$$
\ln \psi(i \lambda)=-\beta \lambda+\frac{\sigma^{2} \lambda^{2}}{2}+\int_{-\infty}^{\infty}\left(e^{-\lambda x}-1\right) G(d x)
$$

где последний интеграл конечен при $\lambda \in(0, a)$. Из (4) и конечности $G((-\infty,-1))$ следует, что при $\lambda \in(0, a)$

$$
\int_{-\infty}^{y} e^{-\lambda x} G(d x) \rightarrow 0 \quad(y \rightarrow-\infty) .
$$

Поскольку при $y<0$ и $\lambda \in(0, a)$

$$
e^{-\lambda y} q(y)=e^{-\lambda y} G((-\infty, y]) \leqslant \int_{-\infty}^{y} e^{-\lambda x} G(d x),
$$

то при $y \rightarrow-\infty$

$$
e^{-\lambda y} q(y) \rightarrow 0 \quad \forall \lambda \in(0, a)
$$

Заметим, что

$$
\begin{aligned}
\int_{-\infty}^{\infty}\left(e^{-\lambda x}-1\right) G(d x) & =\int_{-\infty}^{0}\left(e^{-\lambda x}-1\right) G(d x)+\int_{0}^{\infty}\left(e^{-\lambda x}-1\right) G(d x) \\
& =\int_{-\infty}^{0}\left(e^{-\lambda x}-1\right) d q(x)-\int_{0}^{\infty}\left(e^{-\lambda x}-1\right) d q(x) .
\end{aligned}
$$

Поэтому, интегрируя по частям каждый из последних двух интегралов и учитывая (13), получаем, что

$$
\int_{-\infty}^{\infty}\left(e^{-\lambda x}-1\right) G(d x)=\lambda \bar{q}(\lambda)-\lambda \widehat{q}(\lambda) \quad \forall \lambda \in(0, a),
$$

причем конечность $\bar{q}(\lambda)$ и $\widehat{q}(\lambda)$ гарантируется при $\lambda \in(0, a)$. Соотношение (9) следует из равенств (12) и (14). Лемма 1 доказана.

Доказ а т ель с тв о ле м мы 2. Мы имеем:

$$
g(\lambda)=-\frac{\ln \psi(i \lambda)}{\lambda}=\beta-\frac{\sigma^{2} \lambda}{2}-\bar{q}(\lambda)+\widehat{q}(\lambda)=O(1)+\widehat{q}(\lambda)=O(|\widehat{q}(\lambda)|)
$$

при $\lambda \rightarrow 0_{+}$. Так как $\bar{q}(\lambda)<\infty$ при $\lambda \in(0, a)$, то для произвольного натурального $j$ при $\lambda \rightarrow 0_{+}$

$$
\left|\bar{q}^{(j)}(\lambda)\right|=\int_{-\infty}^{0} x^{j} e^{-\lambda x} q(x) d x \downarrow \int_{-\infty}^{0} x^{j} q(x) d x<\infty .
$$

Поэтому $g^{(j)}(\lambda)=O(1)+\widehat{q}^{(j)}(\lambda)=O\left(\left|\widehat{q}^{(j)}(\lambda)\right|\right)$ при $\lambda \rightarrow 0_{+}$. Лемма 2 доказана.

Лемма 3 доказана в [15, лемма 4.1.5].

Д о к а з а т ел ь с т в о л е м м ы. Мы имеем при $j \in \mathbf{N}$ в силу леммы 2:

$$
\begin{aligned}
(\lambda g(\lambda))^{(j)} & =j g^{(j-1)}(\lambda)+\lambda g^{(j)}(\lambda) \\
& =O\left(\left|\widehat{q}^{(j-1)}(\lambda)\right|\right)+O\left(\left|\lambda \widehat{q}^{(j)}(\lambda)\right|\right)=O\left(\left|\widehat{q}^{(j-1)}(\lambda)\right|\right)
\end{aligned}
$$

при $\lambda \rightarrow 0_{+}$. Утверждение леммы 4 следует из леммы 3 и последнего соотношения. Лемма 4 доказана. 
Лемма 5 есть не что иное, как тауберова теорема 1.6.2 из [15]. Лемма 6 доказывается аналогично теореме 1.3.1 из [15].

Д о к а з а т е л ь с в о л е м м ы 7. Начнем с доказательства утверждения 2). Выберем такую последовательность $t_{k} \uparrow \infty$, что $U\left(\partial A_{k}\right)=0$, где $A_{k}=$ $\left\{x \in \mathbf{R}^{1}:|x| \leqslant t_{k}\right\}$. Пусть $U_{k}^{(m)}$ и $U^{(m)}$ - сужения на $A_{m}$ мер $U_{k}$ и $U$ соответственно. В силу определения слабой сходимости мер, для каждого $m \in \mathbf{N}$ последовательность $U_{k}^{(m)}$ слабо сходится к $U^{(m)}$ при $k \rightarrow \infty$, следовательно (см. [1, раздел VIII.6]),

$$
\int_{A_{m}} e^{-\lambda x} U_{k}(d x) \rightarrow \int_{A_{m}} e^{-\lambda x} U(d x) \quad \forall m \in \mathbf{N}, \quad \lambda \in \mathbf{R}^{1} .
$$

Поэтому при $\lambda \in(a, b)$

$$
\begin{aligned}
\int_{\mathbf{R}^{1}} e^{-\lambda x} U(d x) & =\lim _{m \rightarrow \infty} \int_{A_{m}} e^{-\lambda x} U(d x) \\
& =\lim _{m \rightarrow \infty} \lim _{k \rightarrow \infty} \int_{A_{m}} e^{-\lambda x} U_{k}(d x) \leqslant \limsup _{k \rightarrow \infty}\left(\widetilde{U}_{k}(a)+\widetilde{U}_{k}(b)\right)
\end{aligned}
$$

так как

$$
\begin{aligned}
\int_{A_{m}} e^{-\lambda x} U_{k}(d x) & \leqslant \int_{\mathbf{R}^{1}} e^{-\lambda x} U_{k}(d x) \\
& =\int_{(-\infty, 0)} e^{-\lambda x} U_{k}(d x)+\int_{[0, \infty)} e^{-\lambda x} U_{k}(d x) \\
& \leqslant \int_{(-\infty, 0)} e^{-b x} U_{k}(d x)+\int_{[0, \infty)} e^{-a x} U_{k}(d x) \leqslant \widetilde{U}_{k}(a)+\widetilde{U}_{k}(b) .
\end{aligned}
$$

Для произвольных $k, m \in \mathbf{N}, \lambda \in \mathbf{R}^{1}$ при $B_{m}=\left(-\infty,-t_{k}\right) \cup\left(t_{k}, \infty\right)$

$$
\begin{array}{r}
\left|\widetilde{U}_{k}(\lambda)-\widetilde{U}(\lambda)\right| \leqslant \int_{\left(-\infty,-t_{m}\right)} e^{-\lambda x} U_{k}(d x)+\int_{\left(t_{m}, \infty\right)} e^{-\lambda x} U_{k}(d x) \\
\quad+\int_{B_{m}} e^{-\lambda x} U(d x)+\left|\int_{A_{m}} e^{-\lambda x} U_{k}(d x)-\int_{A_{m}} e^{-\lambda x} U(d x)\right| .
\end{array}
$$

Заметим, что при $h=\lambda-a>0$ и $\lambda \in(a, b)$

$$
\int_{\left(t_{m}, \infty\right)} e^{-\lambda x} U_{k}(d x)=\int_{\left(t_{m}, \infty\right)} e^{-h x-a x} U_{k}(d x) \leqslant e^{-h t_{m}} \widetilde{U}_{k}(a) \longrightarrow 0
$$

при $m \rightarrow \infty$ равномерно по $k \in \mathbf{N}$. Кроме того, при $s=b-\lambda>0$ и $\lambda \in(a, b)$

$$
\int_{\left(-\infty,-t_{m}\right)} e^{-\lambda x} U_{k}(d x)=\int_{\left(-\infty,-t_{m}\right)} e^{s x-b x} U_{k}(d x) \leqslant e^{-s t_{m}} \widetilde{U}_{k}(b) \longrightarrow 0
$$

при $m \rightarrow \infty$ равномерно по $k \in \mathbf{N}$. Зафиксируем $\lambda \in(a, b)$ и $\varepsilon>0$. Выберем такое $m \in \mathbf{N}$, чтобы для произвольных $k \in \mathbf{N}$ выполнялись неравенства

$$
\begin{gathered}
\int_{B_{m}} e^{-\lambda x} U(d x)<\frac{\varepsilon}{4}, \quad \int_{\left(-\infty,-t_{m}\right)} e^{-\lambda x} U_{k}(d x)<\frac{\varepsilon}{4} \\
\int_{\left(t_{m}, \infty\right)} e^{-\lambda x} U_{k}(d x)<\frac{\varepsilon}{4}
\end{gathered}
$$

(это можно сделать согласно соотношениям (16), (18) и (19)). Для полученного $m$ согласно (15) выберем такое $k_{0}$, чтобы при $k \geqslant k_{0}$ было выполнено неравенство

$$
\left|\int_{A_{m}} e^{-\lambda x} U_{k}(d x)-\int_{A_{m}} e^{-\lambda x} U(d x)\right|<\frac{\varepsilon}{4} .
$$


Поэтому из последних четырех неравенств и соотношения (17) следует, что при $k \geqslant k_{0}$

$$
\left|\widetilde{U}_{k}(\lambda)-\widetilde{U}(\lambda)\right|<\varepsilon
$$

что доказывает утверждение 2) леммы 7.

В предположениях пункта 1) леммы 7 для каждого $t>0$ и $\lambda \in(a, b)$

$$
\begin{aligned}
\widetilde{U}_{k}(\lambda) & =\int_{(-\infty, 0)} e^{-\lambda x} U_{k}(d x)+\int_{[0, \infty)} e^{-\lambda x} U_{k}(d x) \\
& \leqslant \int_{(-\infty, 0)} e^{-b x} U_{k}(d x)+\int_{[0, \infty)} e^{-a x} U_{k}(d x) \leqslant \widetilde{U}_{k}(a)+\widetilde{U}_{k}(b)
\end{aligned}
$$

$$
\widetilde{U}_{k}(\lambda) \geqslant \int_{[-t, t])} e^{-\lambda x} U_{k}(d x) \geqslant e^{\lambda t} U_{k}([-t, t]),
$$

откуда следует, что для произвольного фиксированного $t>0$

$$
\sup _{k \in \mathbf{N}} U_{k}([-t, t])<\infty .
$$

Поэтому последовательность мер $\left(U_{k}, k \in \mathbf{N}\right)$ предкомпактна в определенной выше слабой топологии (см. [1, раздел VIII.6, теорема 2]). Иными словами, из всякой ее подпоследовательности можно извлечь сходящуюся. Если подпоследовательность $U_{k(j)}$ слабо сходится к $U$ при $j \rightarrow \infty$ для некоторой меры $U$, то, согласно только что доказанному утверждению 2), при $j \rightarrow \infty$

$$
\widetilde{U}_{k(j)}(\lambda) \rightarrow \widetilde{U}(\lambda) \quad \forall \lambda \in(a, b),
$$

следовательно, $\widetilde{U}(\lambda)=\omega(\lambda)$, и, согласно лемме 6 , у последовательности $U_{k}$ имеется только одна предельная точка $U$ в смысле слабой сходимости, причем $\widetilde{U}(\lambda)=\omega(\lambda)$. Таким образом, $U_{k} \Rightarrow U$ при $k \rightarrow \infty$. Лемма 7 доказана.

Д ок аз а т ель с т в о т е о р е мы 1. Поскольку функция $\ln \psi(t)$ аналитична в полосе $t=\nu+i \lambda\left(\nu \in \mathbf{R}^{1}, \lambda \in(0, a)\right)$ и $\ln \psi(t) \rightarrow 0$ при $t \rightarrow 0$, то для некоторого $b \in(0, a)$ функция

$$
\varphi(t)=\frac{1}{(1-\ln \psi(t))^{r}}
$$

аналитична в полосе $t=\nu+i \lambda\left(\nu \in \mathbf{R}^{1}, \lambda \in(0, b)\right)$. Поэтому, согласно (7), при $\lambda \in(0, b)$

$$
\varphi(i \lambda)=\mathbf{E} e^{-\lambda Y}=\widetilde{P}(\lambda)=\frac{1}{(1-\ln \psi(i \lambda))^{r}},
$$

где $P(d x)$ - распределение случайной величины $Y$. Рассуждая точно так же, как и при доказательстве леммы 1 , получаем из последнего равенства, что при $\eta(\lambda)=$ $-\ln \psi(i \lambda)$ и $\lambda \in(0, b)$

$$
\widetilde{P}(\lambda)=1-\lambda \widehat{T}(\lambda)+\lambda \bar{T}(\lambda)=\frac{1}{(1+\eta(\lambda))^{r}}
$$

(см. обозначения (7)), где $T(x)=\mathbf{P}\{Y>x\}$. Отсюда

$$
\begin{aligned}
\lambda \widehat{T}(\lambda) & =\lambda \bar{T}(\lambda)+\frac{1}{(1+\eta(\lambda))^{r}}=\lambda \bar{T}(\lambda)+\frac{(1+\eta(\lambda))^{r}-1}{(1+\eta(\lambda))^{r}} \\
& =\lambda \bar{T}(\lambda)+\frac{\eta(\lambda) h(\eta(\lambda))}{(1+\eta(\lambda))^{r}}
\end{aligned}
$$

где $\lambda \in(0, b)$ и

$$
h(x)=\frac{(1+x)^{r}-1}{x}=\sum_{j=0}^{\infty} C_{r}^{j+1} x^{j}, \quad|x|<1 .
$$


Согласно (10), $\eta(\lambda)=-\ln \psi(i \lambda)=\lambda g(\lambda)$. Поэтому, поделив (20) на $\lambda$, приходим к соотношению

$$
\begin{aligned}
\widehat{T}(\lambda) & =\bar{T}(\lambda)+\frac{g(\lambda) h(\lambda g(\lambda))}{(1+\lambda g(\lambda))^{r}} \\
& =\bar{T}(\lambda)+g(\lambda) H(\lambda) V(\lambda)=\bar{T}(\lambda)+U(\lambda) V(\lambda)
\end{aligned}
$$

где $\lambda \in(0, b)$ и

$$
\begin{aligned}
& H(\lambda)=h(\lambda g(\lambda)), \quad U(\lambda)=g(\lambda) H(\lambda), \\
& V(\lambda)=v(\lambda g(\lambda)), \quad v(x)=\frac{1}{(1+x)^{r}}, \quad x \geqslant 0 .
\end{aligned}
$$

Дифференцируя (22) $n$ раз по $\lambda$, получаем при $\lambda \in(a, b)$ :

$$
\widehat{T}^{(n)}(\lambda)=\bar{T}^{(n)}(\lambda)+\sum_{j=0}^{n} C_{n}^{j} U^{(j)}(\lambda) V^{(n-j)}(\lambda) .
$$

Мы имеем для произвольного $m \in \mathbf{N}$ :

$$
v^{(m)}(x)=\frac{(-1)^{m} r^{] m[}}{(1+x)^{r+m}} \longrightarrow(-1)^{m} r^{] m[}
$$

при $x \rightarrow 0$, где $r^{] m[}=r(r+1) \cdots(r+m-1)$. Пользуясь формулой $(0.430)$ из [5] для $m$-й производной от сложной функции, получаем, что

$$
\begin{aligned}
V^{(m)}(\lambda) & =\frac{d^{m}}{d \lambda^{m}} v(\lambda g(\lambda)) \\
& =\sum_{i_{1}, \ldots, i_{m}} v^{(k)}(\lambda g(\lambda)) C\left(i_{1}, \ldots, i_{m}\right) \prod_{j=1}^{m}\left((\lambda g(\lambda))^{(j)}\right)^{i_{j}},
\end{aligned}
$$

где суммирование ведется по всем упорядоченным наборам целых неотрицательных чисел $i_{1}, \ldots, i_{m}$, удовлетворяющих условию $\sum_{j=1}^{m} j i_{j}=m$, причем $k=\sum_{j=1}^{m} i_{j}$, а $C\left(i_{1}, \ldots, i_{m}\right)$ - некоторые постоянные. Отсюда с учетом $(25)$ и леммы 4 получаем, что

$$
V^{(m)}(\lambda)=O\left(\left|\widehat{q}^{(m-1)}(\lambda)\right|\right)
$$

при $\lambda \rightarrow 0_{+}$для каждого фиксированного $m \in \mathbf{N}$. Продифференцируем $U(\lambda)$ по $\lambda m$ раз:

$$
U^{(m)}(\lambda)=\frac{d^{m}}{d \lambda^{m}}(g(\lambda) H(\lambda))=\sum_{j=0}^{m} C_{m}^{j} H^{(j)}(\lambda) g^{(m-j)}(\lambda) .
$$

Как и ранее, по формуле для производной от сложной функции,

$$
\begin{aligned}
H^{(m)}(\lambda) & =\frac{d^{m}}{d \lambda^{m}} h(\lambda g(\lambda)) \\
& =\sum_{i_{1}, \ldots, i_{m}} h^{(k)}(\lambda g(\lambda)) C\left(i_{1}, \ldots, i_{m}\right) \prod_{j=1}^{m}\left((\lambda g(\lambda))^{(j)}\right)^{i_{j}},
\end{aligned}
$$

где условия суммирования по $i_{1}, \ldots, i_{m}$, число $k$ и постоянные $C\left(i_{1}, \ldots, i_{m}\right)$ те же, что и в формуле (26). Мы имеем для каждого фиксированного $k \in \mathbf{N}$, что

$$
h^{(k)}(x)=\frac{d^{k}}{d x^{k}}\left(\sum_{j=0}^{\infty} C_{r}^{j+1} x^{j}\right) \longrightarrow C_{r}^{k+1} k !
$$

при $x \rightarrow 0$. С учетом (30) и леммы 4 из (29) получаем, что для произвольного фиксированного $m \in \mathbf{N}$ при $\lambda \rightarrow 0_{+}$

$$
H^{(m)}(\lambda)=O\left(\left|\widehat{q}^{(m-1)}(\lambda)\right|\right)
$$


Из (28), (31) и леммы 2 следует, что

$$
\begin{aligned}
U^{(m)}(\lambda) & =H(\lambda) g^{(m)}(\lambda) \sum_{j=1}^{m} C_{m}^{j} H^{(j)}(\lambda) g^{(m-j)}(\lambda) \\
& =(r+o(1)) g^{(m)}(\lambda)+O\left(\sum_{j=1}^{m}\left|\widehat{q}^{(j-1)}(\lambda) \widehat{q}^{(m-j)}(\lambda)\right|\right)
\end{aligned}
$$

при $\lambda \rightarrow 0_{+}$, так как $h(x) \rightarrow r$ при $x \rightarrow 0$ (см. формулу (21)). Поэтому, согласно лемме 3 ,

$$
U^{(m)}(\lambda)= \begin{cases}(r+o(1)) \widehat{q}^{(m)}(\lambda), & \text { если } I_{m}=\infty, \\ O\left(\left|\widehat{q}^{(m)}(\lambda)\right|\right), & \text { если } I_{m}<\infty,\end{cases}
$$

при $\lambda \rightarrow 0_{+}$, так как если $I_{m}=\infty$, то

$$
\begin{aligned}
g^{(m)}(\lambda) & =\frac{d^{m}}{d x^{m}}\left(\beta+\frac{\sigma^{2} \lambda}{2}+\widehat{q}(\lambda)-\bar{q}(\lambda)\right) \\
& =O(1)+\widehat{q}^{(m)}(\lambda)=(1+o(1)) \widehat{q}^{(m)}(\lambda) .
\end{aligned}
$$

Согласно лемме 3 , из соотношений $(24),(32)$ и (33) для каждого фиксированного $n \in \mathbf{N}$ такого, что $I_{n}=\infty$, мы имеем:

$$
\begin{aligned}
\widehat{T}^{(n)}(\lambda) & =\bar{T}^{(n)}(\lambda)+U^{(n)}(\lambda) V(\lambda)+\sum_{j=0}^{n-1} C_{n}^{j} U^{(j)}(\lambda) V^{(n-j)}(\lambda) \\
& =O(1)+(r+o(1)) \widehat{q}^{(n)}(\lambda)+O\left(\sum_{j=0}^{n-1}\left|\widehat{q}^{(j)}(\lambda) \widehat{q}^{(n-j-1)}(\lambda)\right|\right) \\
& =O(1)+(r+o(1)) \widehat{q}^{(n)}(\lambda)+o\left(\widehat{q}^{(n)}(\lambda)\right)=(1+o(1)) r \widehat{q}^{(n)}(\lambda)
\end{aligned}
$$

при $\lambda \rightarrow 0_{+}$. Согласно лемме 5 , отсюда получаем, что $T(t) \stackrel{w}{\sim} r q(t)$ при $t \rightarrow \infty$. Теорема 1 доказана.

Автор выражает глубокую признательность рецензенту за ценные замечания.

\section{СПИСОК ЛИТЕРАТУРЫ}

1. Антонов C. Н., Круглов В. М. Об асимптотическом поведении безгранично делимых распределений в банаховом пространстве. - Теория вероятн. и ее примен., 1982 , т. 27 , в. 4 , с. $625-642$.

2. Антонов С.Н., Круглов В.М. Еще раз об асимптотическом поведении безгранично делимых распределений в банаховом пространстве. - Теория вероятн. и ее примен., 1984 , т. 29 , в. 4 , с. $735-742$.

3. Buldygin V.V., Klesov O.I., Steinebach J. G. Properties of a subclass of Avakumović functions and their generalized inverses. - Ukrainian Math. J., 2002, v. 54, № 2, p. 179-206.

4. Buldygin V.V., Klesov O.I., Steinebach J.G. On factorization representations for Avakumović-Karamata functions with nondegenerate groups of regular points. Anal. Math., 2004, v. 30, №3, p. 161-192.

5. Градитейн И. С., Рьюик И. М. Таблицы интегралов, сумм, рядов и произведений. М.: Физматгиз, 1962, 1108 с.

6. Золотарев В. М. Об асимптотическом поведении одного класса безгранично делимых законов распределения. - Теория вероятн. и ее примен., 1961, т. 6, в. 3, c. $330-334$.

7. Janković $S$. Enlargement of the class of geometrically infinitely divisible random variables. - Publ. Inst. Math. (Beograd) (N.S.), 1993, v. 54 (68), p. 126-134. 
8. Петров В. В. Суммы независимых случайных величин. М.: Наука, 1985.

9. Клебанов Л. Б., Мания Г. М., Меламед И. А. Одна задача В. М. Золотарева и аналоги безгранично делимых и устойчивых распределений в схеме суммирования случайного числа случайных величин. - Теория вероятн. и ее примен., 1984, т. 29 , в. 4 , с. $757-760$.

10. Круглов В.М., Королев В.Ю. Предельные теоремы для случайных сумм. М.: Изд-во Моск. ун-та, 1990, 269 с.

11. Sato K.-I. Lévy Processes and Infinitely Divisible Distributions. Cambridge: Cambridge Univ. Press, 1999, 486 p.

12. Сеибнев M. С. Асимптотика безгранично делимых распределений в $\mathbf{R}^{n}$. - Труды Ин-та математики СО АН СССР, 1989, т. 13, с. 100-116.

13. Сенета E. Правильно меняющиеся функции. М.: Наука, 1985, 141 с.

14. Феллер В. Введение в теорию вероятностей и ее приложения, т. 2. М.: Мир, 1984, $752 \mathrm{c}$.

15. Якымив А.Л. Вероятностные приложения тауберовых теорем. М.: Физматлит, $2005,256 \mathrm{c}$.

Поступила в редакцию 22.IX.2008

(c) 2010 г.

ЯСЬКОВ П. А.*

\section{СИЛЬНАЯ СХОДИМОСТЬ КРАТНЫХ СУММ НЕОРТОГОНАЛЬНЫХ СЛУЧАЙНЫХ ВЕЛИЧИН ${ }^{1)}$}

Получены новые достаточные условия п.н. сходимости кратных рядов из неортогональных случайных величин и новый вариант усиленного закона больших чисел. Данные условия описаны в виде ограничений на вторые моменты, а также на верхние грани средних попарных произведений рассматриваемых величин. Наши теоремы улучшают как хорошо известные результаты для квазистационарных полей, так и недавние, связанные с обобщением свойства квазистационарности.

Ключевые слова и фразы: случайное поле, усиленный закон больших чисел, сходимость рядов почти наверное, зависимые случайные величины.

1. Введение. В настоящей статье рассматривается вопрос об асимптотическом поведении кратных сумм, образованных элементами случайного поля определенного класса. Точнее говоря, исследуются условия применимости усиленного закона больших чисел, а также сходимости кратных рядов с вероятностью единица. Упомянутый класс весьма широк, в частности, он содержит квазистационарные поля.

Данному вопросу посвящено много работ. Исчерпывающие ответы получены для следующих типов случайных полей: составленных из независимых одинаково распределенных величин (см. [1] и [2]), ортогональных (см. [3] и [4]), однородных (см. [5]), квазистационарных (см. [6]). Ограничения общего вида, не преполагающие какой-либо структуры зависимости, рассмотрены в [7]. Наша статья мотивирована недавними работами [8] и [9], где допускается некоторое отклонение от свойства квазистационарности.

Многие результаты о квазистационарных случайных величинах получаются с помощью максимальных неравенств Морица (см. [10]). В нашем случае эти неравенства

* Московский государственный университет им. М.В. Ломоносова, Ленинские горы, 119991 Москва, ГСП-1, Россия; e-mail: yaskov_pavel@mail.ru

1) Работа выполнена при финансовой поддержке РФФИ, грант № 07-01-00373. 